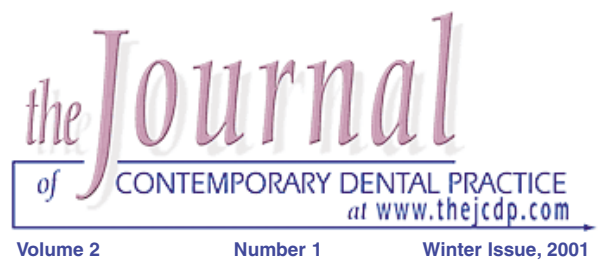

\title{
Four-Handed Dentistry: Instrument Transfer
}

\section{Betty Ladley Finkbeiner, CDA, RDA, MS}

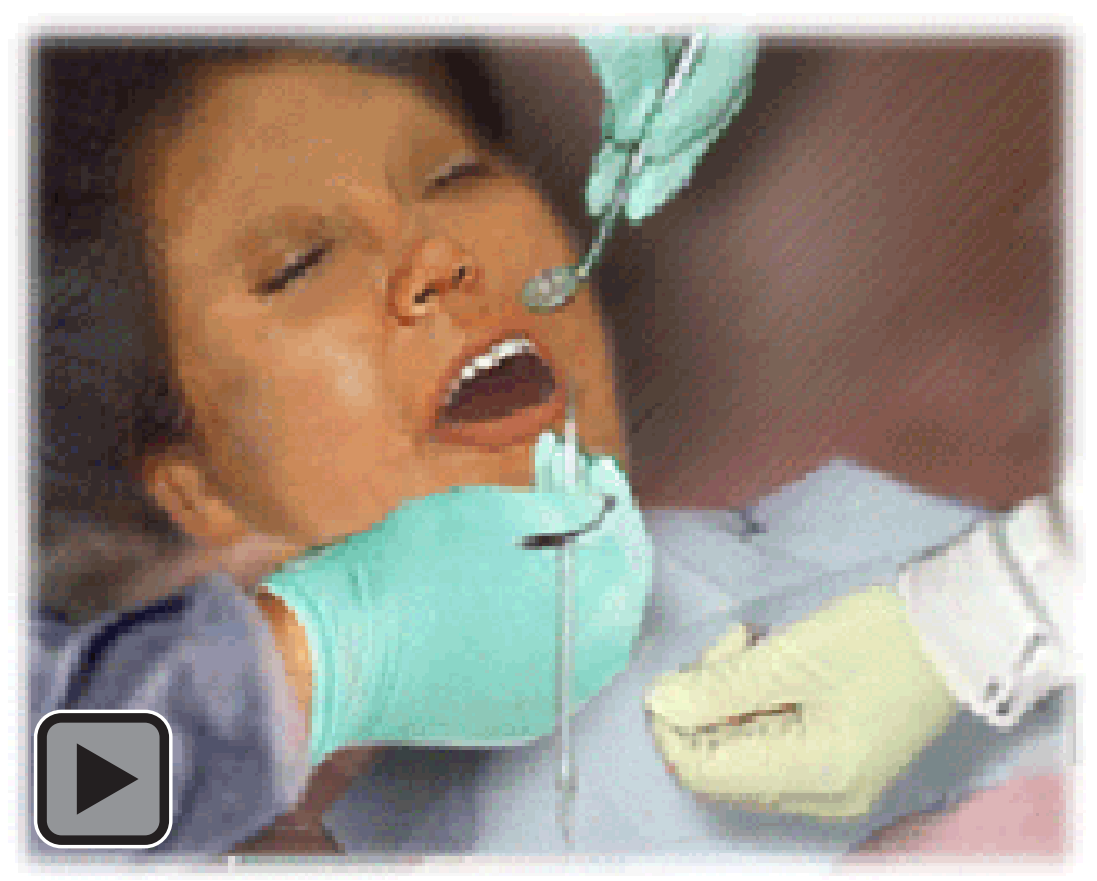

Abstract

The efficient exchange of instruments between the operator and the dental assistant is fundamental to the facilitation of an efficient and stress-free dental practice. This requires a commitment on the part of the members of the operating team to specific work practices before and during a dental procedure. The objectives of this article include the description of team member responsibilities during an instrument transfer and the identification of the benefits of a proper instrument transfer technique. Objectives also include specific instrument grasps and descriptions of transfer methods that require practice on the part of the operating team in order to attain proficiency.

Keywords: Instrument transfer, single-handed transfer, two-handed transfer, hidden syringe transfer, pen grasp, modified pen grasp, palm grasp, palm thumb/thumb-to-nose grasp.

(c) Seer Publishing 
Introduction

Instrument transfer is the process of transferring instruments and materials to and from the operator, within the transfer zone, at a precise moment of need. (Figure 1) An important pre-requisite to successful instrument transfer is the ability of each member of the dental team to understand the procedure and anticipate each other's needs. Use of an efficient instrument transfer is one of the basic skills every dental team must learn in order to be productive and stress free. This notion may seem basic, yet many dentists and assistants struggle to refine a smooth productive technique.

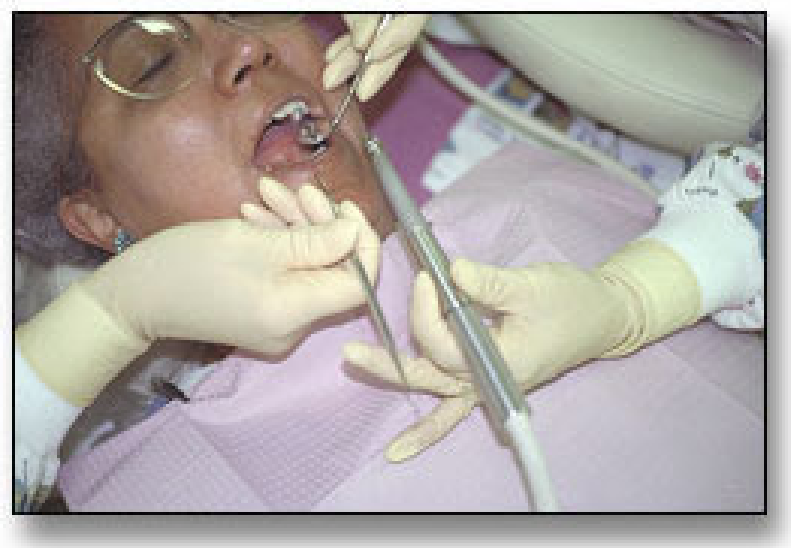

Figure 1

During the past twenty-five years there has been a diminished emphasis on formal education in the basic principles of the four-handed dentistry concept including the use of efficient instrument transfer techniques. This has resulted in dentists not learning proper ergonomic strategies in dental school, or following graduation due to fewer continuing education courses being offered on the subject. These dentists tend to work more inefficiently and engage in more time consuming movements during dental procedures than is necessary. Proper instrument transfer techniques serve to conserve motion, increase the flow of the procedure and reduce eyestrain by eliminating the need to look away from the brightly illuminated operative field during the transfer. It is advisable to re-evaluate the instrument transfer techniques used during a dental procedure in order to ensure safety during the transfer and ensure that the benefits of a proper technique (listed below) are realized.
- The operator is able to maintain vision on the operative field thus reducing eyestrain associated with changing light intensity and focal length accommodation.

- The operating team conserves time and motion during instrument transfers.

- There is a reduction in stress and strain on the operating team due to the uninterrupted flow of the procedure without the nagging delays associated with locating and delivering instruments if an orderly system is not used.

- When instrument transfer is used in conjunction with the oral evacuator and the air/water syringe, the operative site will always be clean and the next instrument will be ready for use.

- Percutaneous injuries associated with use of dental instruments can be minimized using a prescribed transfer technique.

\section{Team Responsibilities During Instrument Transfer}

\section{Basic Principles}

Successful instrument transfer is predicated on a set of principles that require organization and planning in advance. The first step in this process is to work from a well-defined treatment plan so that instruments and materials required for a planned procedure can be prepared in advance. Dental assistants should thoroughly understand the procedure to be performed in order to anticipate the sequence in which the instruments and materials will be used. With this knowledge the dental team can develop a standardized routine for the performance of most dental procedures.

Additional strategies such as the delegation of all material preparation and instrument transfer to the assistant as well as the delegation of expanded/advanced functions to legally qualified assistants will maximize the use of the dentist's time. Using ergonomically designed equipment and placing the patient in a supineposition improves access and visibility for the operating team. (Figure 2) Placing supportive equipment and supplies within a 21 -inch radius of the assistant's hands as well as confining movements to the oral cavity and the adjacent transfer zone over the 


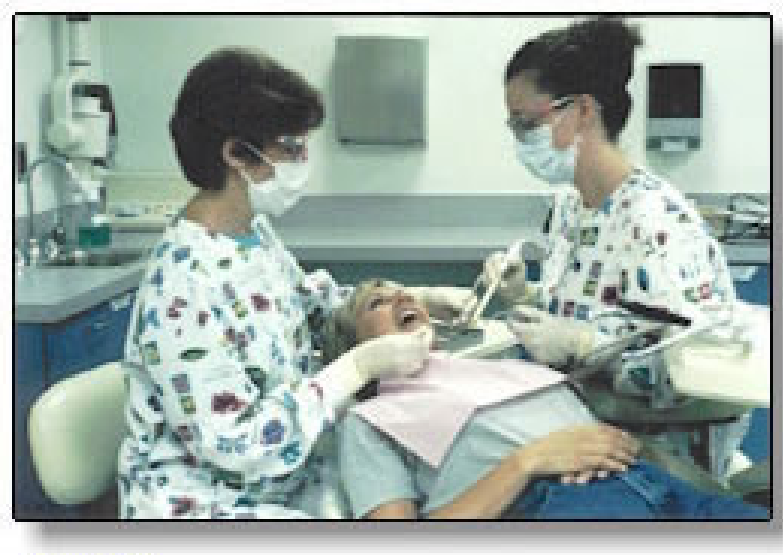

Figure 2

patient's chest will result in the conservation of time and motion during the procedure.

To ensure a successful instrument transfer, each member of the team must assume specific, but often related responsibilities.

\section{Operator Requirements}

In order to maximize the efficiency of an instrument transfer technique the operator should maintain a finger rest for his/her working hand in the oral cavity so that the actual location of the transfer between team members will be predictable. Such predictability is essential for smooth, safe transfers to occur.

A non-verbal signal to indicate a need to exchange an instrument is helpful in order to avoid tedious and repetitious verbal communication throughout the workday. It is not uncommon for the number of instrument exchanges during a typical restorative procedure to exceed one hundred. After the non-verbal signal is given, the operator needs to place the used instrument in his/her hand in a position that enables the assistant to safely retrieve it and transfer the new instrument. A simple withdrawal of the used instrument from the operative field can serve as a non-verbal signal and it repositions the used instrument for better access by the dental assistant. (Figure 3)

To reduce eyestrain the operator needs to maintain his/her eyes on the operative field and refrain from removing instruments from or replacing instruments onto the pre-set tray. By making an instrument exchange predictable there is no need

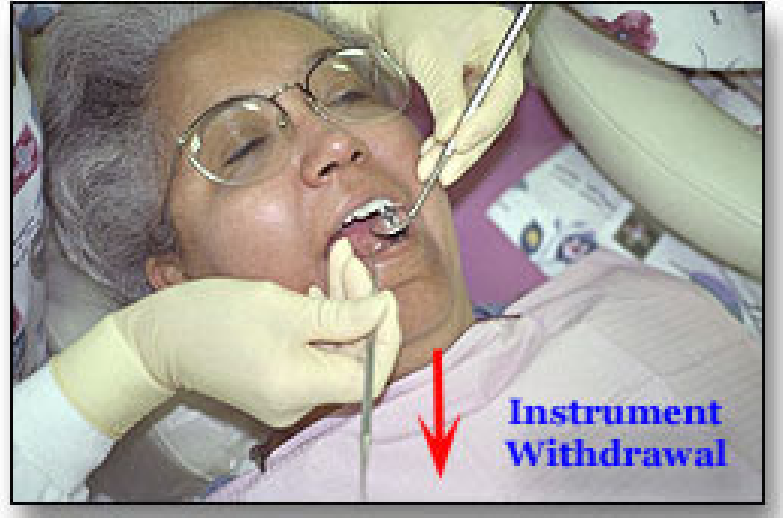

Figure 3

for the operator to look away from the oral cavity during the procedure.

\section{Assistant Requirements}

In order to maximize the efficiency of an instrument transfer technique the assistant needs to maintain instruments on a pre-set instrument tray in the sequence of their use in order to facilitate rapid location of needed instruments during a procedure. (Figure 4) The assistant also needs to anticipate the need for the next instrument and stay alert for any change in the procedure to be ready to modify the sequence of instruments when necessary.

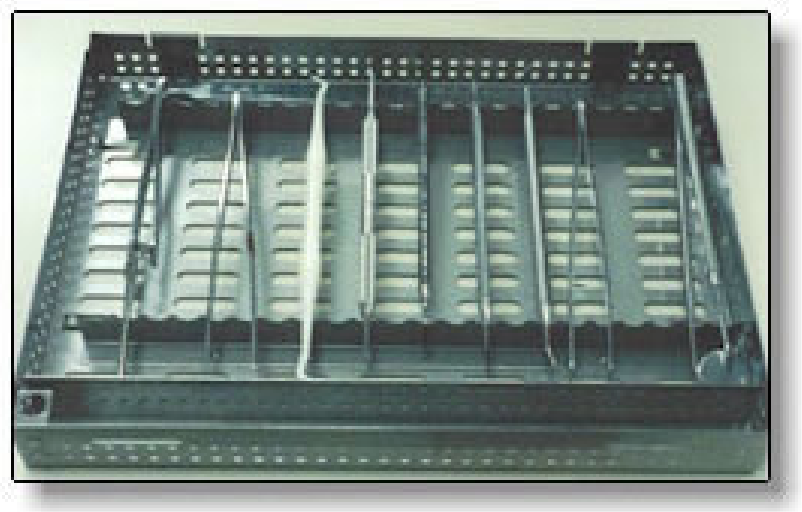

\section{Figure 4}

As the instrument transfer begins, the working ends of instruments need to be positioned for the proper dental arch; up for the maxilla and down for the mandible. During the transfer the assistant needs to use positive pressure to ensure the operator senses that the instrument has been 
delivered; this eliminates the operator from looking away from the site, or fumbling the delivered instrument. Immediately after the transfer debris from the used instrument should be removed before returning it to the tray or to the operator for use again.

\section{Team Requirements}

In order to maximize efficiency and safety during an instrument transfer it is advisable for the operating team to observe patient movement, especially during the exchange of an anesthetic syringe or other sharp instruments.

Following a safe standardized transfer procedure should be used to avoid injuries. Safe techniques include the maintenance of firm control of the instrument at all times, exchanging instruments only in the transfer zone over the patient's chest and avoiding unpredictable movements during the transfer. Laying instruments or material on the patient's napkin should be avoided to prevent injury.

\section{Instrument Grasps}

The three most common methods used by an operator to hold an instrument are the pen grasp, modified pen grasp and palm grasp.

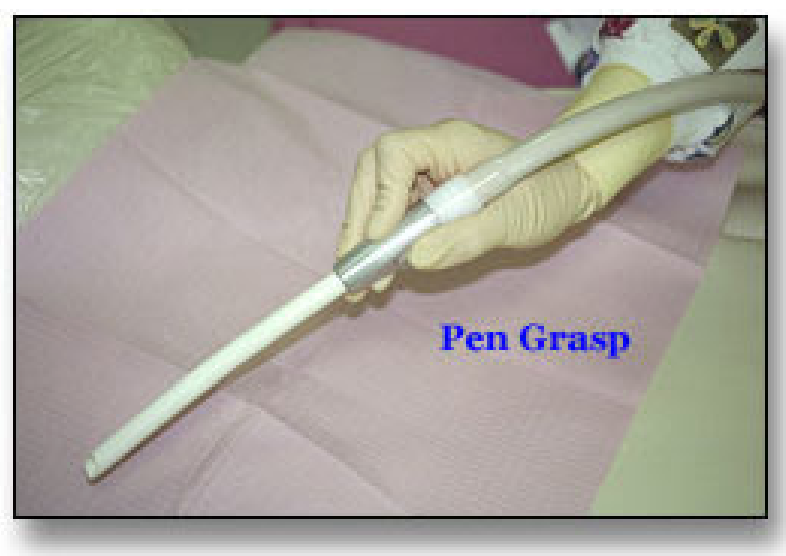

Figure 5

- The pen grasp resembles the position commonly used to hold a pen or pencil and is widely used for most operative instruments. (Figure 5)

- The modified pen grasp is similar to the pen grasp except the operator uses the pad of the middle finger on the handle of the instrument. Some operators prefer

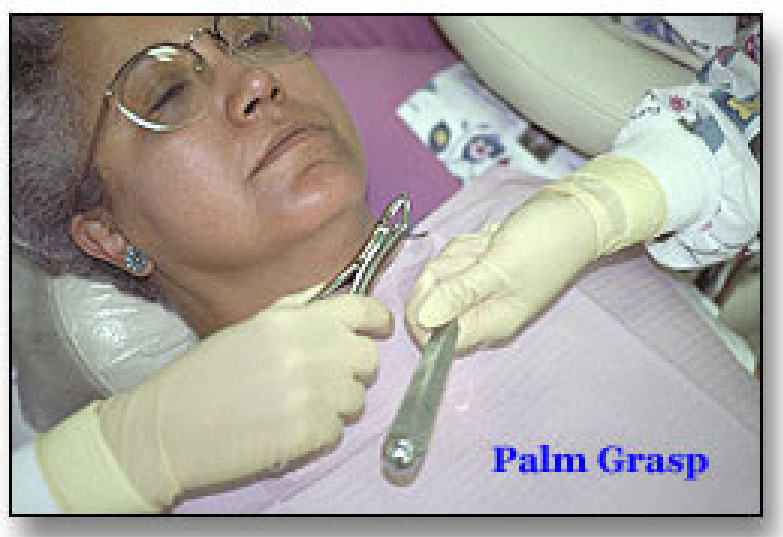

Figure 6

this method since they feel it provides more strength and stability in some procedures.

- The palm grasp is used for bulky instruments. It is commonly used for surgical forceps, rubber damp clampforceps, straight chisels and the air/water syringe. (Figure 6)

- The palm-thumb, or thumb-to-nose grasp is used by the assistant for holding the oral evacuator. The operator may use this with instruments that require a more vertical movement. (Figure 7)

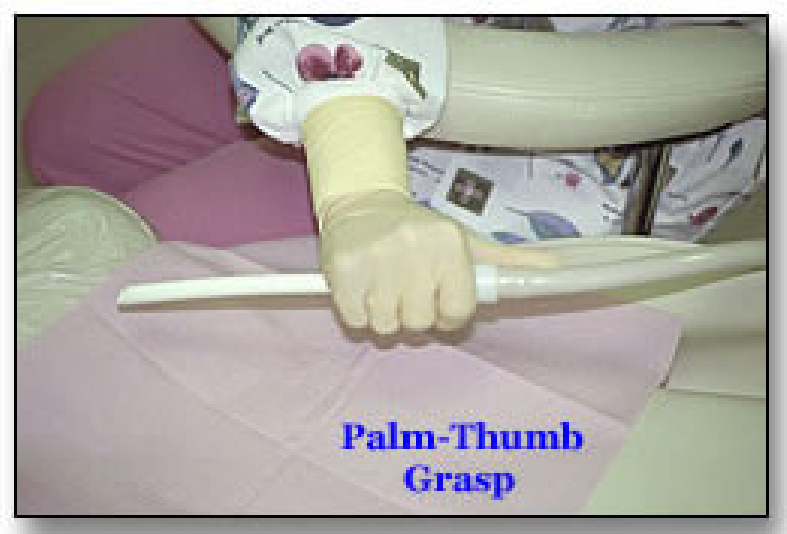

Figure 7

Types of Instrument Transfer

The three most common instrument transfers used today in dentistry are the single-handed, two-handed and hidden syringe transfers.

\section{Single-handed Transfer Technique}

(Right-handed operator)

The single-handed transfer is used during most common treatment procedures. (Figure 8) This 


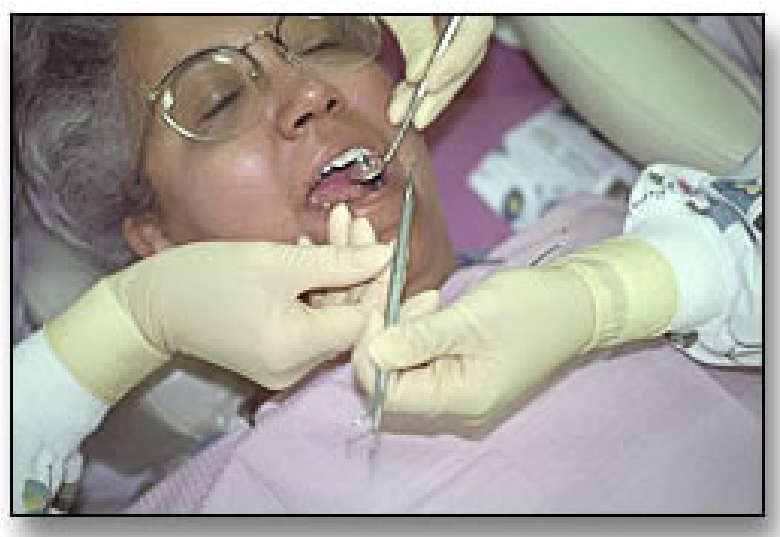

Figure 8

procedure requires that the assistant transfer instruments with the left hand and hold the oral evacuator tip and air/water syringe in the right hand. When working with a left-handed operator all the positions described here are reversed.

The assistant's hand is divided into two parts: the pick up and the delivery portions. For practical understanding the following discussion defines the fingers of the hand as the thumb, first, second, third and fourth. Some assistants receive the used instrument with only the small finger. This action poses a potentialstability problem and two fingers should be used for retrieval when possible.

Some assistants are tempted to flip or twirl the retrieved instrument back into the delivery portion of the hand. This can pose danger to the patient if the instrument suddenly flips out of the hand. When the used instrument is received from the operator in the pick up portion of the hand, the assistant can roll the instrument back into position by pressing the tip of the thumb against the handle of the retrieved instrument and rolling it into the delivery position.

The single handed instrument transfer with a righthanded operator is illustrated in the following procedural outline.

- Assemble instruments in sequence of use and place the instrument tray as close to the patient as possible. The tray may be positioned in a vertical or horizontal position. (Figure 9)

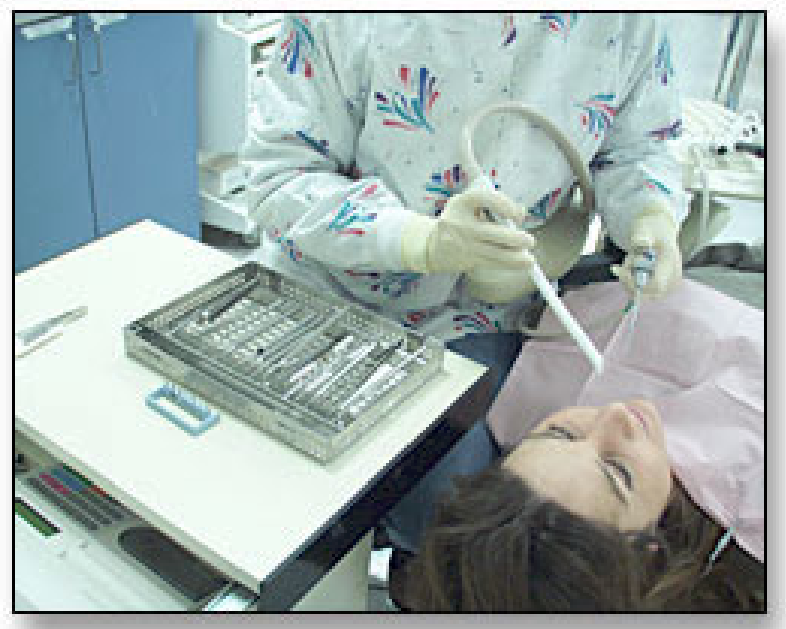

Figure 9

- Place auxiliary equipment such as the anesthetic syringe, or rubber dam on the mobile cabinet farthest from the patient. At the beginning of the procedure simultaneously pass the mirror with the right hand and the explorer with the left hand. (Figure 10)

- $\quad$ Pick up the instrument to be transferred in the left hand and position it between the first finger and thumb at the nonworking third of the instrument.

(Figure 11)

- Rest the instrument on the middle finger, making certain that the working end is positioned for the correct arch and position it within 10-12 inches from the operator's hand in readiness for a transfer when needed. (Figure 12)

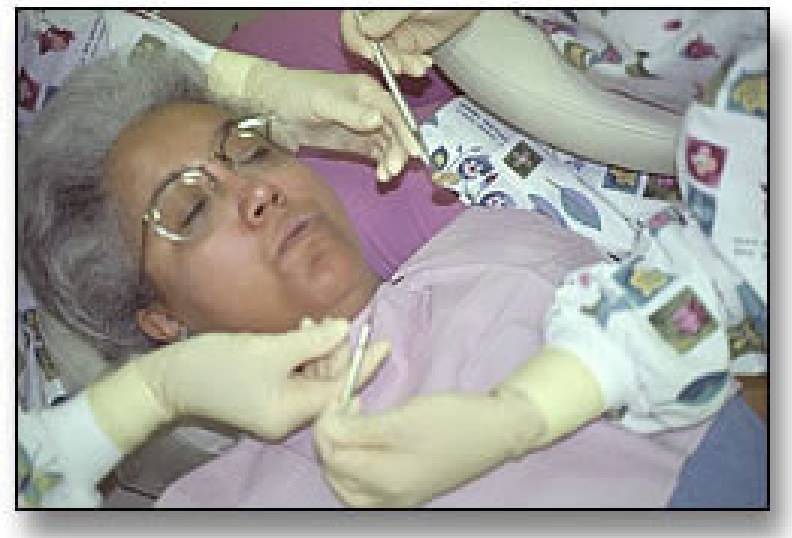

Figure 10 


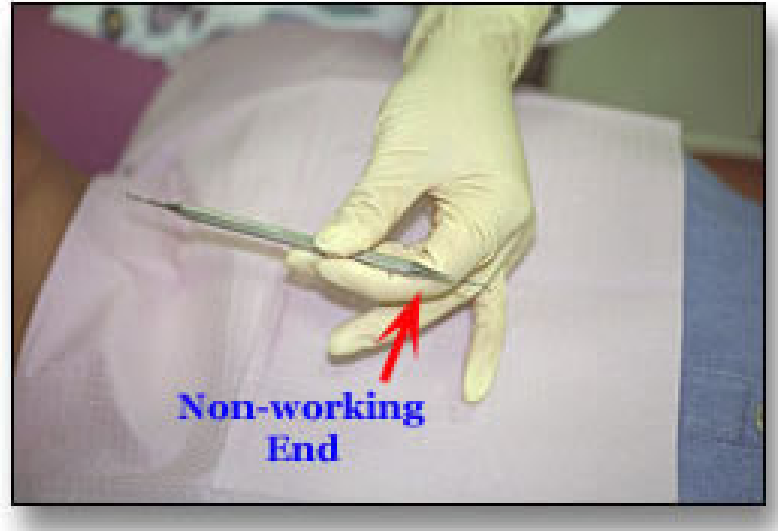

Figure 11

- The operator signals for an exchange by moving the instrument being used from the tooth and bringing it outside the mouth. When possible, a finger rest may be maintained. (Figure 13)

- The assistant grasps and tucks the used instrument toward the wrist with the pick up portion of the hand. (Figure 14)

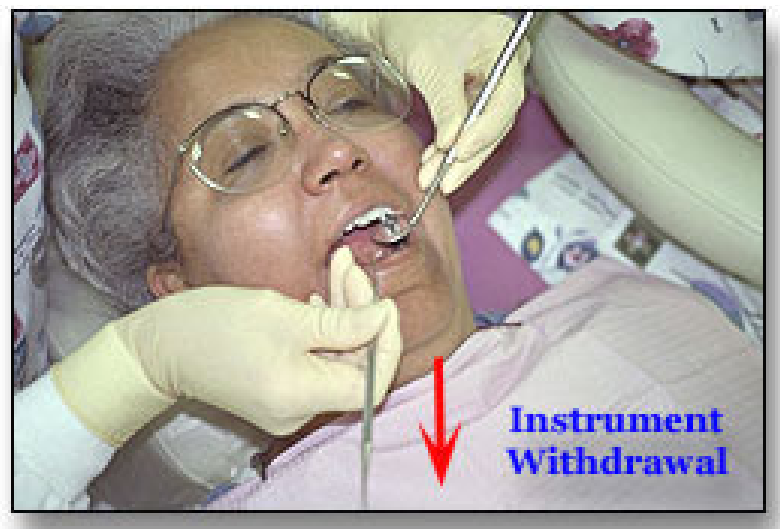

Figure 13

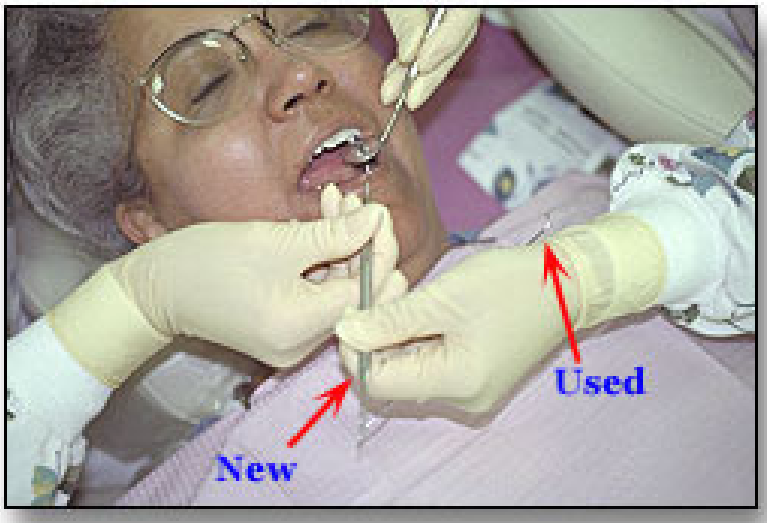

Figure 15

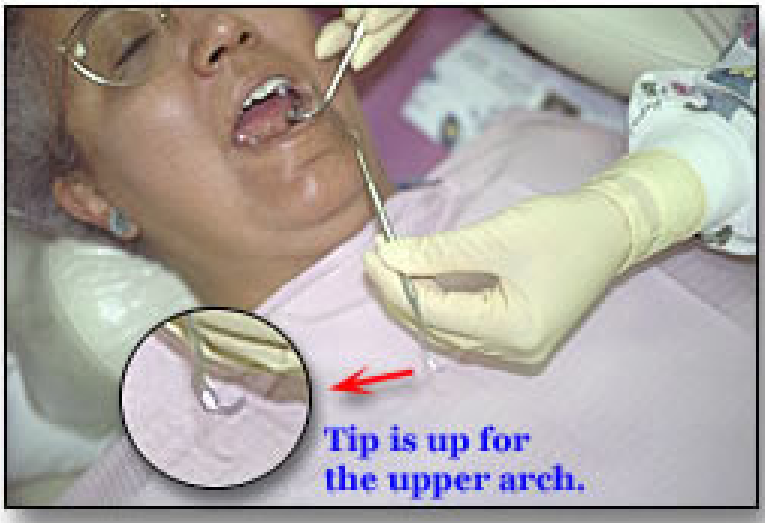

Figure 12

- The new instrument is delivered into the operator's hand with the delivery portion of the hand and the operator returns to the mouth with the new instrument. (Figure 15)

- With the thumb, the assistant rolls the instrument from the palm up to the ring finger until it is above the first knuckle. Take care to avoid puncturing the gloves. (Figure 16)

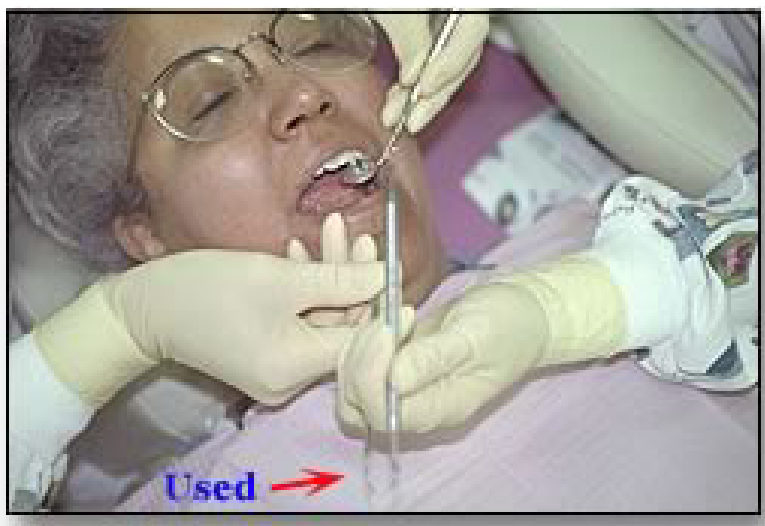

Figure 14

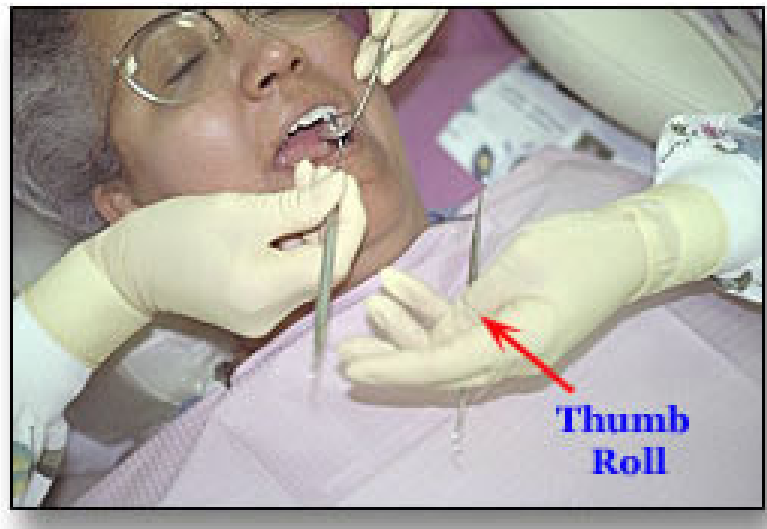

Figure 16

6

The Journal of Contemporary Dental Practice, Volume 2, No. 1, Winter Issue, 2001 


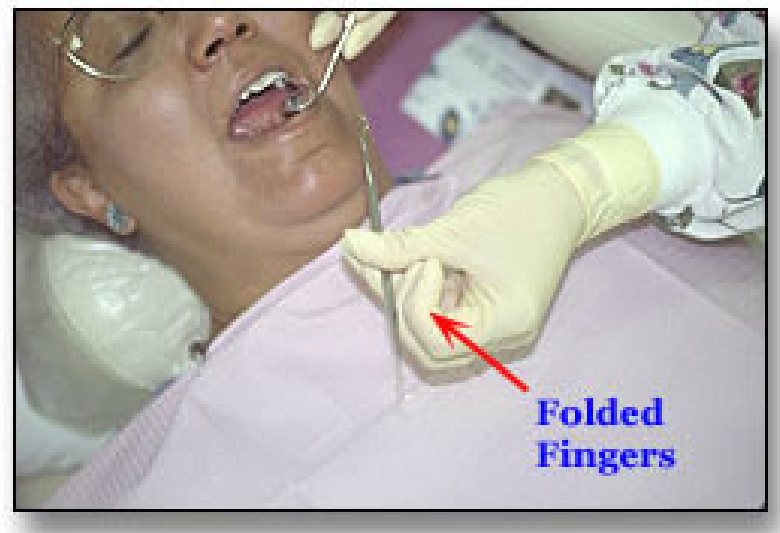

Figure 17

- Fold the index and middle fingers under the handle and return the instrument to the holding position. (Figure 17)

- If the instrument is not to be used again, it can be returned to the proper position on the tray.

- When the air-water syringe and the oral evacuator are used, the assistant places the air-water syringe in the right hand to free the other for the instrument transfer. (Figure 18)

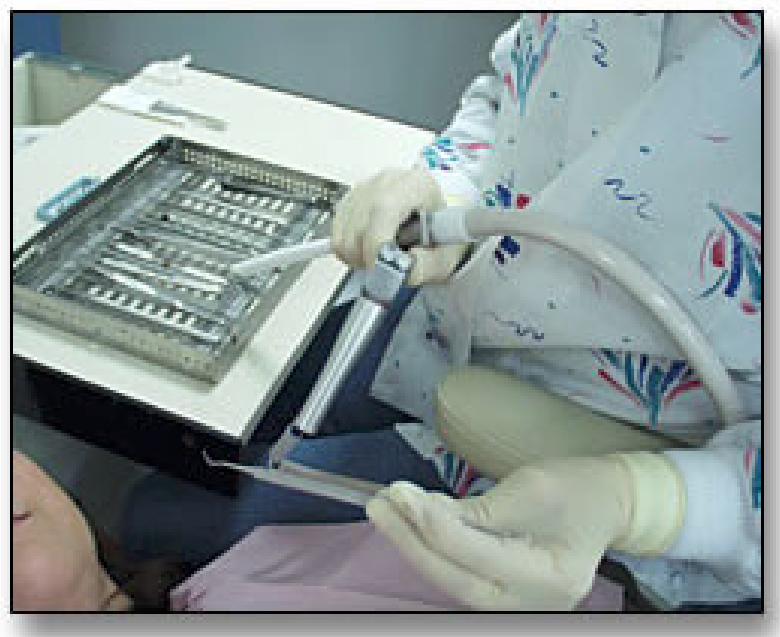

Figure 18

Bulky instruments can be transferred in the same single handed exchange as described above. Many dental units, such as the split unit, prohibit effective handpiece transfer since the handpiece is outside the 21 -inch radius of the assistant's hand. This unit placement leads to decreased effectiveness in terms of time and motion. When a transthorax unit is used and handpieces are within the transfer zone, the assistant should then utilize the following procedure:

- The handpiece is made parallel with the instrument to be exchanged. (Figure 19)

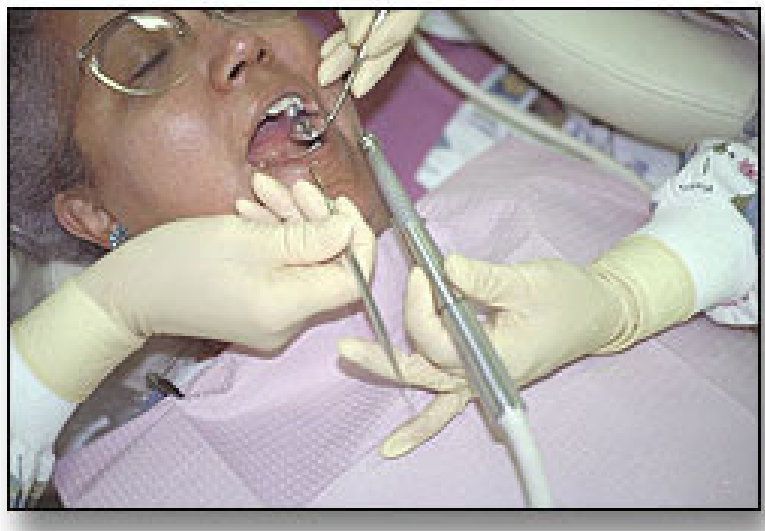

Figure 19

- $\quad$ The return of the handpiece in the pick up portion of the assistant's hand is done in the same manner as any other instrument even though it is bulkier. For this reason, the two fingers used in the pick up method provide greater stability. (Figure 20)

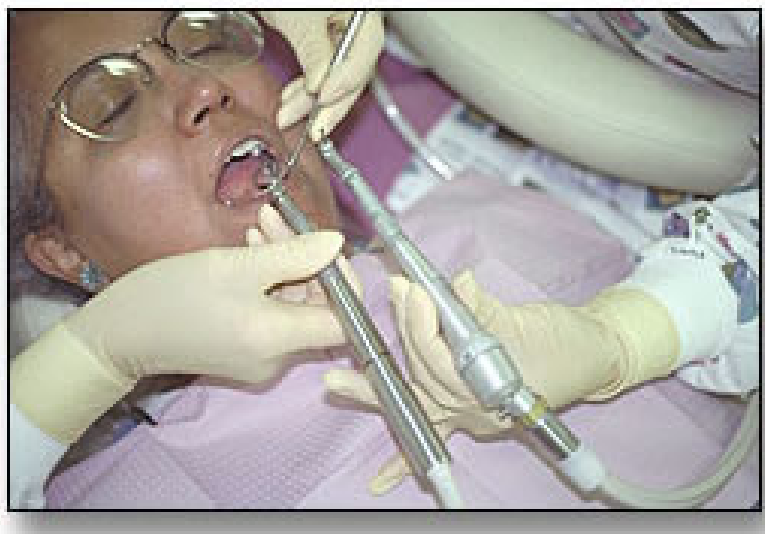

Figure 20

- The handpiece is then returned to the dental unit

\section{The Two-Handed Transfer}

The two-handed transfer is used when transferring bulky instruments such as the rubber dam clamp forceps or surgical forceps. (Figure 21) This transfer requires the assistant to pick up the used instrument with one hand and deliver the new instrument with the opposite hand. This 


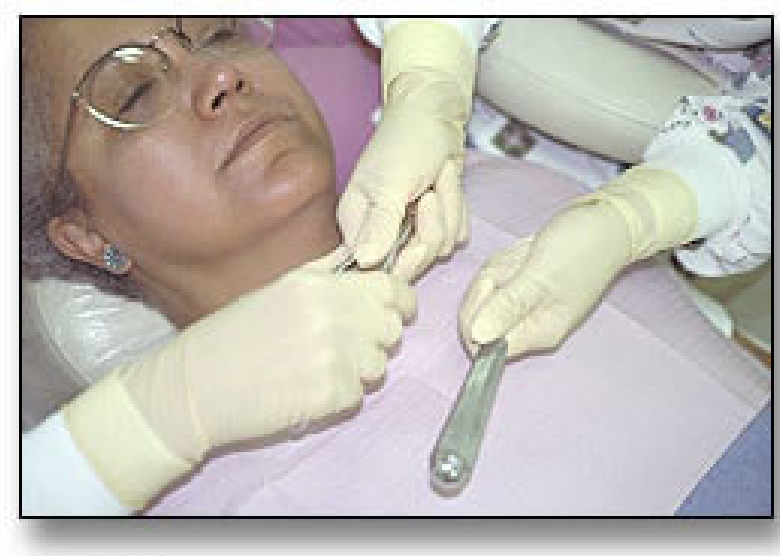

Figure 21

exchange requires more movement and limits the use of the HVE and air-water syringe.

\section{Hidden Syringe Transfer}

The hidden syringe transfer is named as such because it takes place out of view under the chin of the patient. It enables the operator to receive the anesthetic syringe safely and out of the patient's line of sight thus avoiding undue patient stress. This transfer also occurs within the transfer zone.

This transfer requires that the assistant and operator plan in advance the technique to avoid the potential of a needle-stick. Often the operator prefers to transfer the instrument behind the patient. This area is outside the transfer zone, causing the assistant to use a Class $V$ movement and violate safe transfer. This transfer can be adapted to the use of wand type anesthesia very effectively. Care should be taken that the base unit is located nearby the assistant for easy access. Using a traditional anesthetic syringe the following steps may be followed:

- A $2 \times 2$ gauze is passed to dry the site. Topical anesthetic may be applied with a cotton-tipped applicator if desired.

(Figure 22)

- The protective cap on the needle is loosened slightly. (Figure 23)

- The syringe is held in the assistant's right hand when assisting a right handed operator while the assistant stabilizes the operator's hand using a firm grasp. (Figure 24)

- The operator positions the right hand upright with the index, and middle fingers

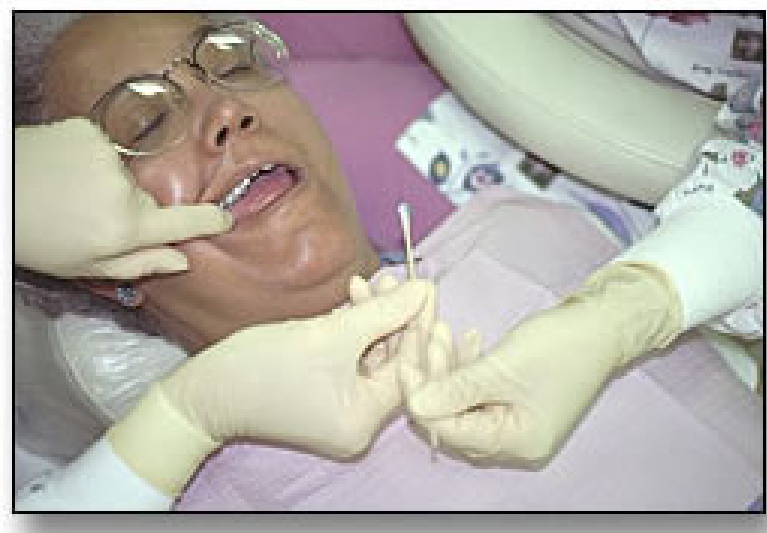

Figure 22

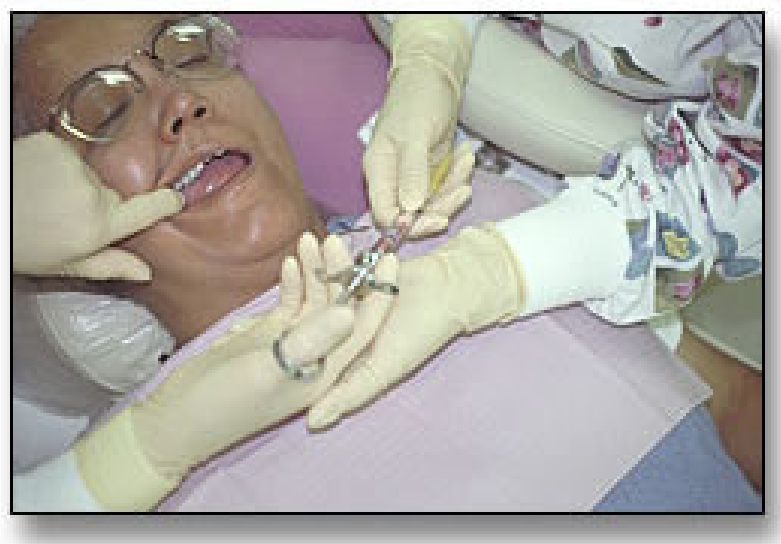

Figure 23

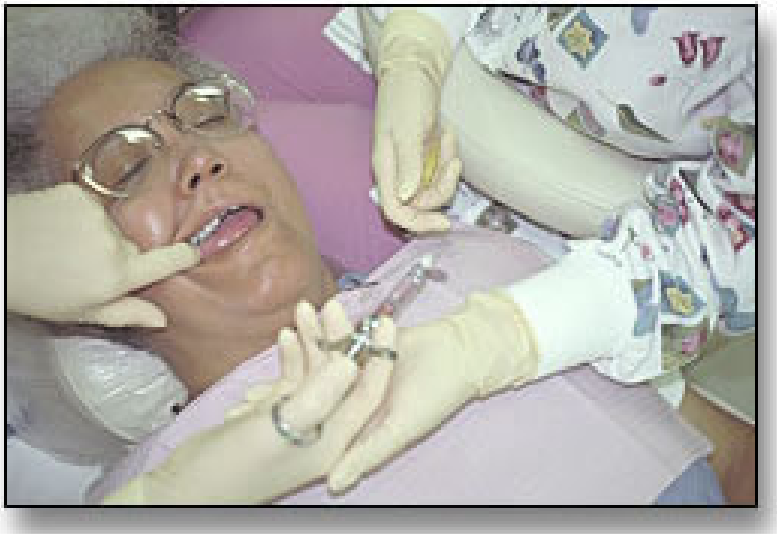

Figure 24

extended along with the thumb to receive the syringe. While the assistant holds the operator's right hand firmly the thumb ring of the syringe is positioned over the operator's thumb and lowered to rest between the awaiting index and middle fingers.

- The assistant's hand carefully removes the protective cover while maintaining the firm grasp of the operator's right hand to 
avoid any inadvertent movement. Once the cap is removed and the assistant's right hand is away from the exposed needle the operator's hand can be released to signal that the syringe is ready for use.

- The cover is placed into the recapping device while the operator administers the anesthetic. (Figure 25) If the patient is a small child, the assistant can gently place his or her arms over the child to prevent a sudden movement that could result in injury during the injection procedure.

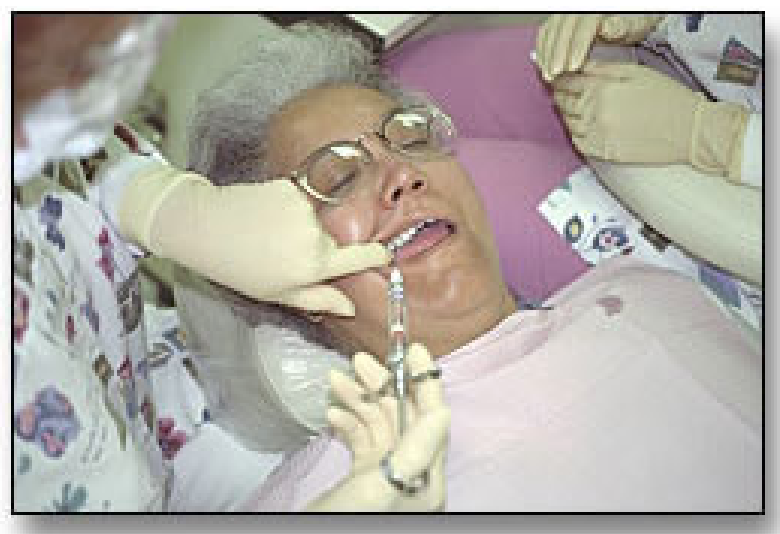

Figure 25

- The retrieval of the syringe is similar to the delivery in that, using the left hand, the assistant grasps the operator's right hand upon withdrawal of the syringe from the patient's mouth to hold it in a predictable position. The assistant then grasps the syringe by the barrel to avoid contacting the contaminated needle. A gauze sponge is exchanged for the syringe to use as a compress over the injection site. (Figure 26)

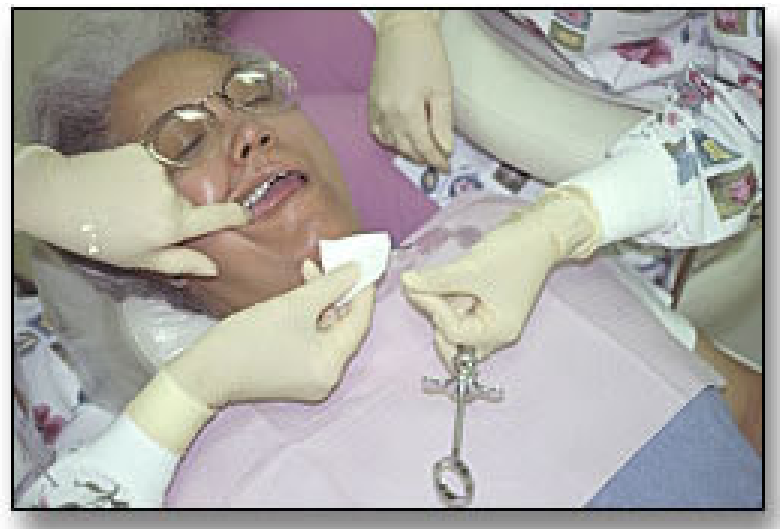

Figure 26
- The syringe is placed in a recapping device. (Figure 27) Though it requires a movement out of the operator's zone, some operators prefer to replace the syringe in the recapping device to avoid a potential accident.

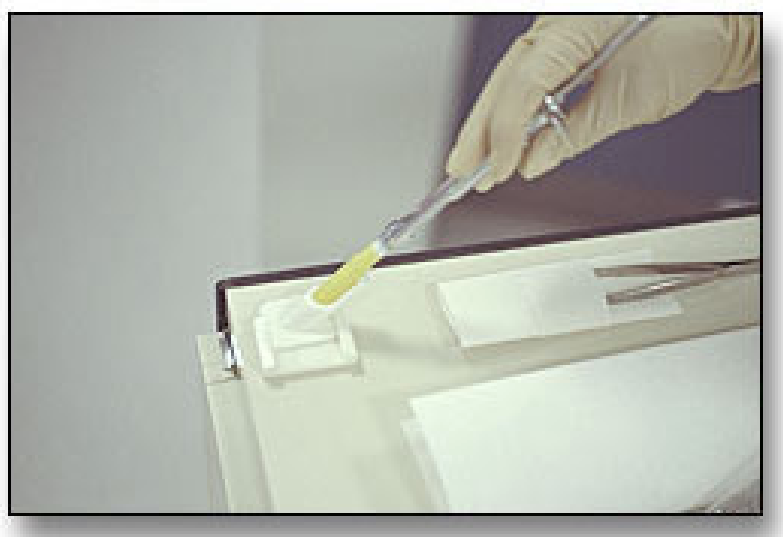

Figure 27

- The mouth may be rinsed at this time if any anesthetic was dropped on the tongue.

\section{Special Instruments or Situations}

There will be times or situations that dictate modification of the single-handed transfer. The following suggestions may aid during these times.

\section{Delivery of the Dental Mirror and Explorer (Figure 28 A \& B)}

- These two instruments are transferred simultaneously by the assistant at the beginning of most dental procedures.

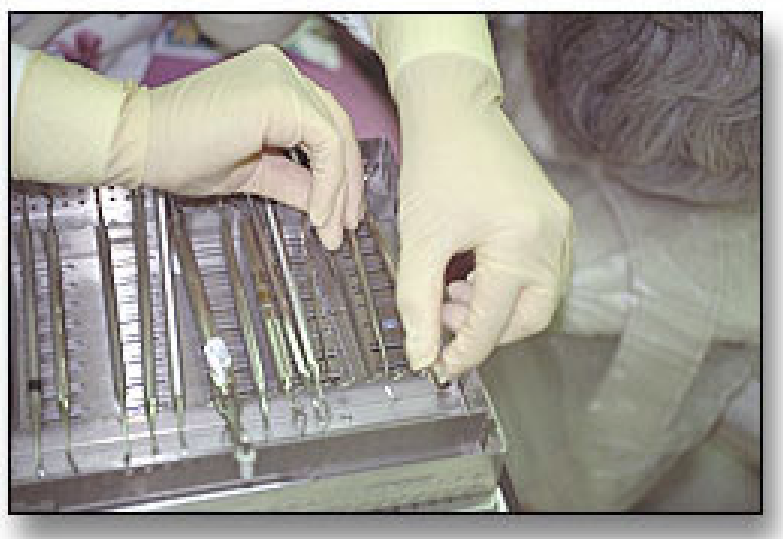

Figure 28A 


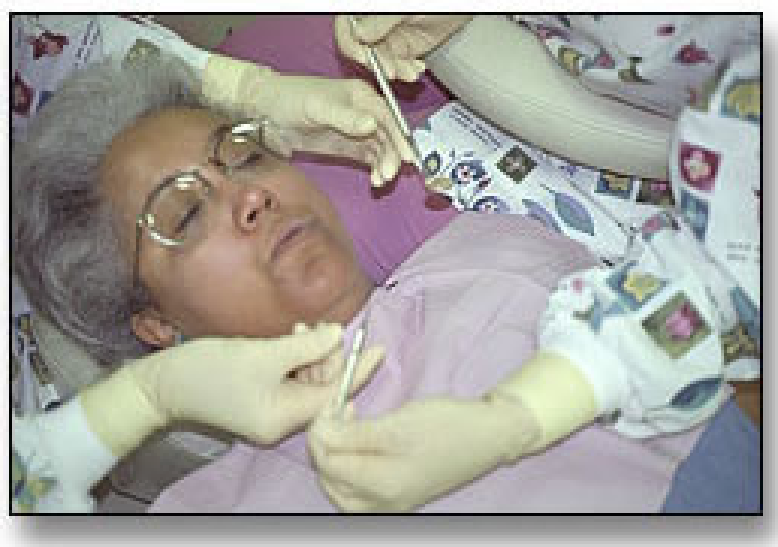

Figure 28B

- The dental mirror is picked up by the handle end using the right hand. At the same time the explorer is picked up with the left hand at the one third of the instrument handle nearest the assistant.

- Position the instruments in the delivery portion of the hands and when the operator signals, pass in the same manner as described in the section above.

- The mirror can be retrieved with the right hand at the conclusion of the procedure.

\section{Use of Non-Locking Tissue Forceps}

(Figure $29 \mathrm{~A}-\mathrm{C}$ )

- If non-locking forceps are used, the assistant must maintain a grasp on the forceps to ensure the beaks do not separate during transfer.

- After the material to be transferred has been placed into the forceps beaks the forceps are paralleled with the used instrument that is to be exchanged.

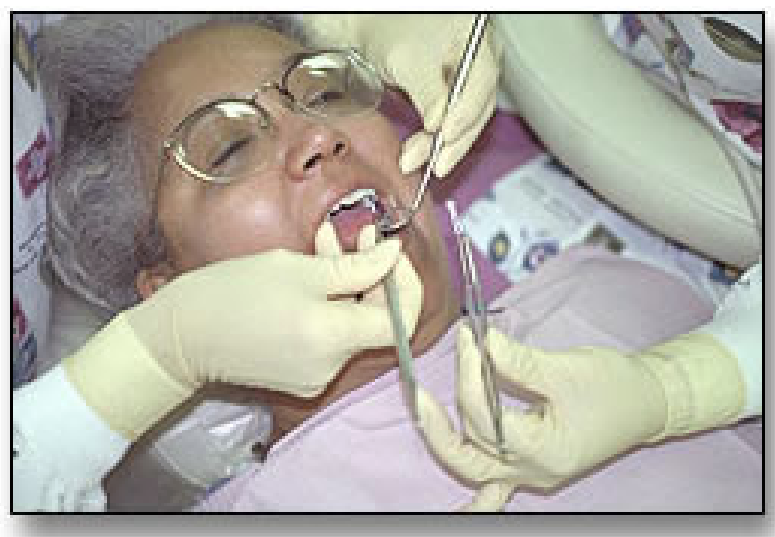

Figure 29A

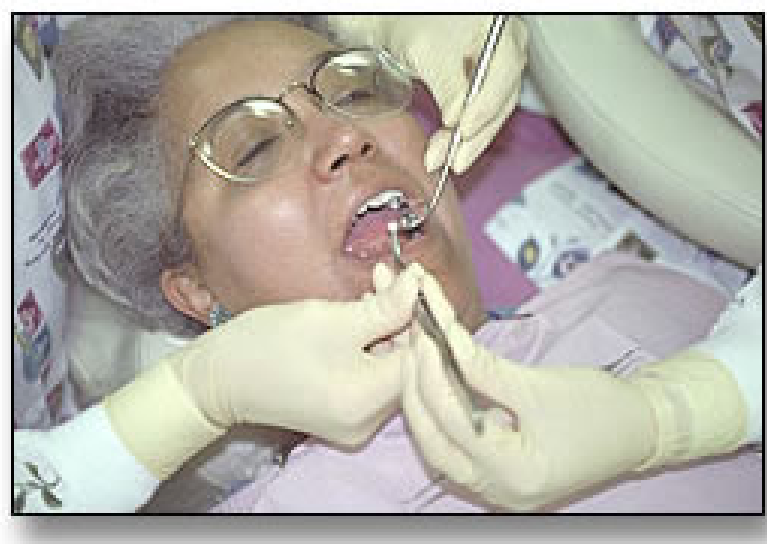

Figure 29B

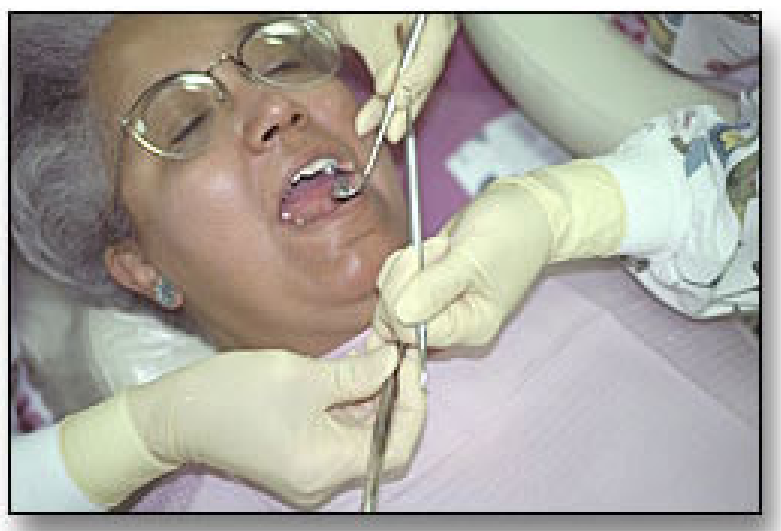

Figure 29C

- The instrument is exchanged in the same manner as other instruments.

- When the forceps are returned to the assistant, the working end of the forceps is grasped in the palm of the hand to eliminate dropping the contents.

- The forceps are not rolled back into position, but rather the assistant discards the materials from the forceps and returns the instrument to the tray.

\section{Delivery of Small Items}

(Figure 30 A \& B)

- Small items such as a cotton applicator can be passed to the operator as any other instrument.

- Medicaments can be passed by first passing the insertion instrument and then holding the pad with the medicament in the transfer zone for easy access to the operator. 


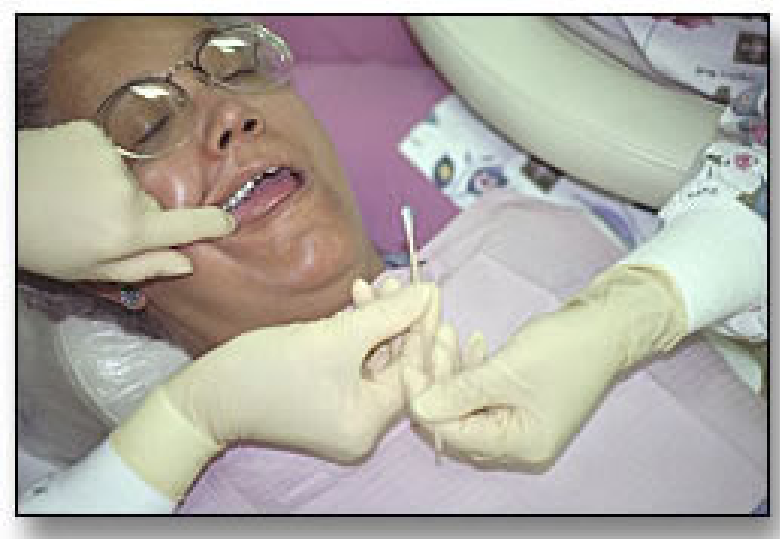

Figure $30 \mathrm{~A}$

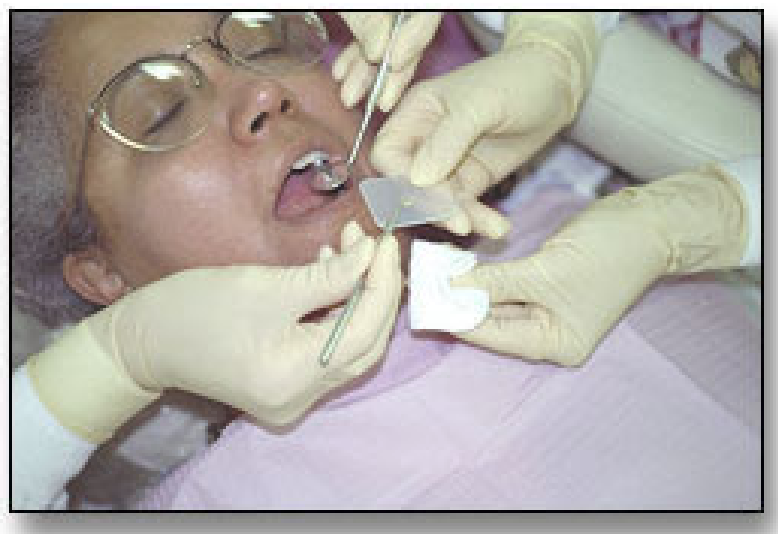

Figure $30 \mathrm{~B}$

\section{Delivery of Scissors \\ (Figure $31 \mathrm{~A}-\mathrm{C}$ )}

- The assistant picks up the scissors from the tray with the left hand; opens the handles slightly and parallels the scissors with the instrument to be exchanged.

- The operator modifies the hand position by placing the thumb and first or second finger into the rings of the handle.

- The scissors are returned with the beaks pointing toward the assistant.

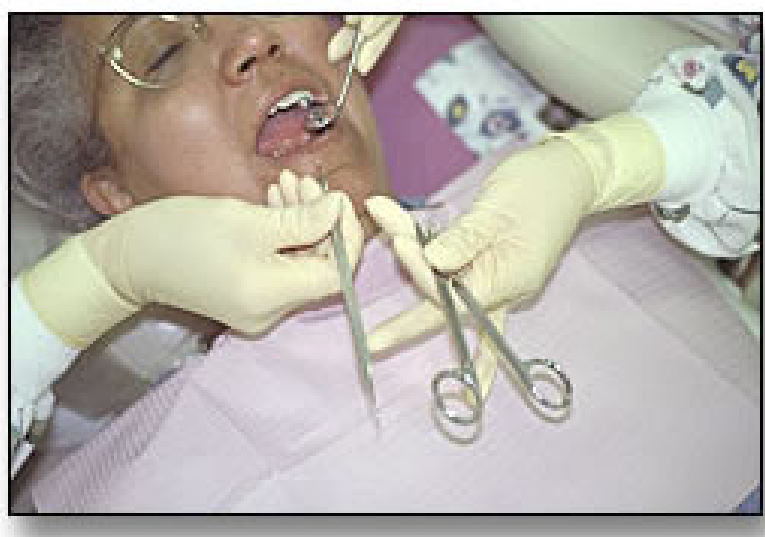

Figure 31A

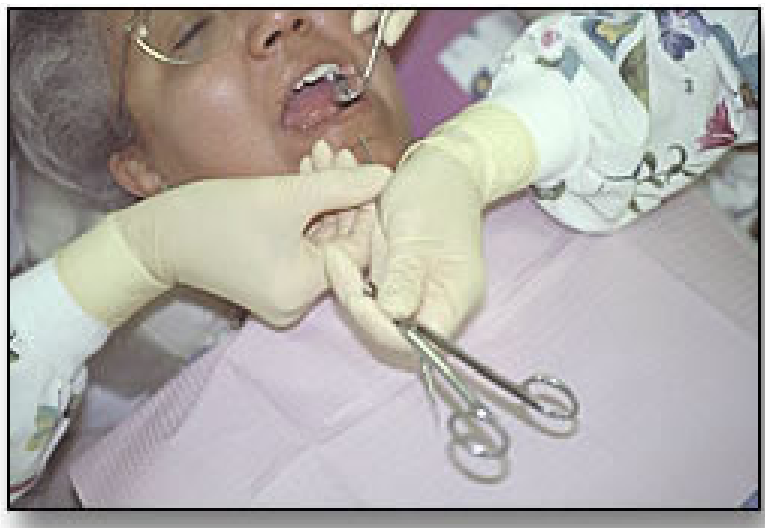

Figure 31B

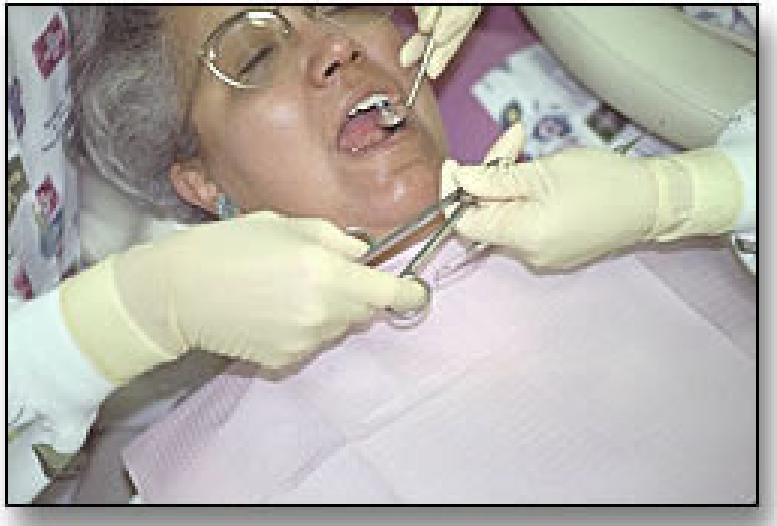

Figure $31 \mathrm{C}$

\section{Conclusion}

Use of standardized instrument transfer techniques can yield significant benefits for the operating team. Like most psychomotor skills, practice is required before proficiency can be achieved. The effort required is worthwhile if the team wants to maximize efficiency, safety and reduce stress throughout the workday. 


\section{References}

1. Bramson JB, Smith S, Rogagnoli G. Evaluating dental of fice ergonomic. Risk factors and hazards. J Am Dent Assoc. February 1998;129(2): 174-83.

2. Finkbeiner BL. Four Handed Dentistry: A Handbook of Clinical Application and Ergonomic Concepts, Upper Saddle River, NJ, Prentice Hall, 2001.

3. Guay A H. Commentary: ergonomically related disorders in dental practice. J Am Dent Assoc. February 1998, 129(2):184-6. No abstract available.

4. Hunk K. Ergonomics: a case study in preventing repetitive motion injuries. Journal Dental School Technology, June 1996;13(5): 35-7.

5. Liskiewicz ST, Kerschbaum, WE. Cumulative trauma disorders: an ergonomic approach for prevention. J Dent Hyg 1997 Summer;71(4): 162-7. Review.

6. Murphy, DC. Ergonomics and the Dental Health Care Worker. Washington, DC American Public Health Association, 1998.

7. Robinson, GE, et al. Four-handed Dentistry: The Whys and Wherefores. J Am Dent Assoc 1968 Sep;77(3): 573-9.

\section{Betty Ladley Finkbeiner, CDA, RDA, MS}

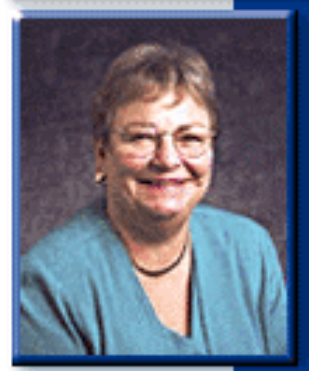

Betty Ladley Finkbeiner is the Chairperson of the Dental Assisting Program at Washtennaw Communtiy College, Ann Arbor, Michigan. She has served as a consultant and staff representative for the American Dental Association's (ADA) Commission on Dental Accreditation and as a consultant to the Dental Assisting National Board. In 1999 she was appointed to the Michigan Board of Dentistry.

Ms Finkbeiner has authored articles in professional joumals and co-authored several textbooks including: Practice Management for the Dental Team, Comprehensive Dental Assisting: A Clinical Approach, and Review of Comprehensive Dental Assisting. Most recently she has authored a handbook with Prentice Hall entitled, Four-handed Dentistry: A handbook of Clinical Application and Ergonomic Concepts. She has co-authored videotape productions including Medical Emergencies for the Dental Team, Four-handed Dentistry: An Ergonomic Concept, and Infection Control for the Dental Team.

Correspondence related to this article can be sent to her at the following address:

Whastenaw Community College

4800 E. Huron River Drive

Ann Arbor, MI 48106

e-mail: blf@wccnet.org 